\title{
Heterogeneity of tumor chemosensitivity in ovarian epithelial cancer revealed using the adenosine triphosphate-tumor chemosensitivity assay
}

\author{
JIN ZHANG and HONGXIA LI \\ Department of Obstetrics and Gynecology, Beijing Shijitan Hospital, Capital Medical University, \\ Haidian, Beijing 100038, P.R. China
}

Received April 22, 2014; Accepted January 19, 2015

DOI: $10.3892 / 01.2015 .3056$

\begin{abstract}
Ovarian cancer has a poor prognosis, primarily due to the heterogeneity in chemosensitivity among patients. In the present study, this heterogeneity was evaluated in ovarian epithelial cancer (OEC) using an in vitro adenosine triphosphate tumor chemosensitivity assay (ATP-TCA). Specimens were collected from 80 patients who underwent cytoreductive surgery. Viable ovarian cancer cells obtained from malignant tissues were tested for sensitivity to paclitaxel (PTX), carboplatin (CBP), topotecan (TPT), gemcitabine (GEM), docetaxel (TXT), etoposide, bleomycin and 4-hydroperoxycyclophosphamide using ATP-TCA. The sensitivity, specificity, positive predictive value and negative predictive value for the clinical chemotherapy sensitivity of OEC were 88.6, 77.8, 83 and $84.8 \%$, respectively. PTX demonstrated the highest sensitivity of all agents tested $(82.5 \%$ in all specimens, $85.7 \%$ in recurrent specimens), followed by CBP (58.8 and $60.7 \%$, respectively). The sensitivities to PTX and docetaxel $(\mathrm{P}<0.001)$ were correlated, in addition to those of CBP, TPT and GEM $(\mathrm{P}<0.001)$. Early-stage (I/II) and high- to mildly-differentiated OEC specimens revealed a lower chemosensitivity than advanced-stage (III) or low-differentiated specimens, respectively. The present study indicated that ATP-TCA is an effective method for guiding the choice of chemotherapy drugs. Notable heterogeneity of chemosensitivity was observed in the OEC specimens.
\end{abstract}

\section{Introduction}

Ovarian cancer has one of the highest mortality rates among all gynecological malignancies (1). Paclitaxel plus platinum

Correspondence to: Professor Hongxia Li, Department of Obstetrics and Gynecology, Beijing Shijitan Hospital of Capital Medical University, 10 Tieyi Road, Haidian, Beijing 100038, P.R. China

E-mail: lihx6260@gmail.com

Key words: adenosine triphosphate tumor chemosensitivity assay, heterogeneity, in vitro, ovarian cancer therapy is rapidly gaining acceptance as the standard clinical chemotherapy regimen for ovarian cancer, and is highly effective as a first-line therapy for patients with advanced ovarian malignancies. However, the majority of patients still experience relapse within a short time following chemotherapeutic intervention $(2,3)$.

The failure of chemotherapy may be associated with the heterogeneity of tumor tissues, one of the primary characteristics of malignant tumors. Previous studies have demonstrated that tumors originate from cancer stem cells (4). However, the continuous differentiation of stem cells during the process of tumor growth results in daughter cells with variations in their genetic and molecular characteristics following several rounds of proliferation, leading to differences in tumor growth, invasiveness, drug susceptibility and prognosis (5). Variation in the genotype or subtype of cells among patients results in ovarian cancer heterogeneity, which affects the efficacy of chemotherapy agents $(6,7)$.

Evaluating the heterogeneity of tumor tissues is essential for predicting the chemosensitivity of tumors prior to beginning a chemotherapy regimen. The adenosine triphosphate-tumor chemosensitivity assay (ATP-TCA) is an in vitro drug sensitivity testing method that has become widely used for determining the drug sensitivity rates of solid tumors in recent years (8-10). This method has notable advantages for guiding individual treatments and chemotherapy protocol design, and evaluating novel chemotherapy drugs. In particular, ATP-TCA has been used for $>20$ years in research on ovarian cancers. Sevin et al (11) first used ATP-TCA to evaluate the chemosensitivity of ovarian cancer cell lines and tissues in 1988, obtaining high correlations between in vitro drug sensitivity and in vivo patient response. Since then, a number of studies have analyzed the high sensitivity, specificity and clinical relevance of ATP-TCA in ovarian cancers (12-14). In recurrent ovarian cancer, ATP-TCA could be used to guide the selection of chemotherapy drugs and potentially improve the clinical response rate and survival of patients $(15,16)$.

When using the ATP-TCA protocol, it is necessary to ensure an optimized method of calculation for interpreting the results. In previous studies, the following parameters were determined by analyzing the correlation between doses of 
various drugs and the rate of inhibition: Calculation of the area under the chemotherapy drug dose-inhibition curve (AUC) using the trapezoidal rule, comparison of drug concentrations that achieve either 50 or $90 \%$ growth inhibition in vitro $\left(\mathrm{IC}_{50}\right.$ and $\mathrm{IC}_{90}$, respectively), and the sensitivity index (SI), which is calculated by adding the percentage of tumor growth inhibition (TGI) at each concentration tested (17). Konecny et al (12) analyzed three ATP-TCA parameters and observed that the SI was superior to the $\mathrm{AUC}$ or $\mathrm{IC}_{50}$ for the interpretation of test results. Several studies suggested that an SI of $>250$ was the best standard to predict chemoresistance $(13,14)$. The present study therefore selected 250 as the cut-off point of SI and further proved this hypothesis.

In the current study, the ATP-TCA method was used to assess the heterogeneity of chemosensitivity in ovarian epithelial cancer (OEC), and the correlation between the clinical features of tumors and chemosensitivity was analyzed to gain further insight into the heterogeneity of OECs and provide an additional rationale for the use of ATP-TCA technology in guiding clinical treatment to potentially improve patient outcomes.

\section{Materials and methods}

Ethics statement. The study was approved by the Ethics Committee of the Beijing Shijitan Hospital of Capital Medical University (Beijing, China). Written informed consent was obtained from all the patients and families prior to surgery. All procedures were conducted in accordance with the Code of Ethics of the World Medical Association (Declaration of Helsinki, 1964, as revised in 2004).

Tumor specimens. A total of 80 fresh tumor specimens were obtained from patients who had OEC and underwent surgery at the Beijing Shijitan Hospital, Beijing University People's Hospital and People's Liberation Army General Hospital, China, between April 2012 and February 2013. Routine histopathological analysis was performed for samples obtained from the same tissues to determine the stage and histological features of the tumor samples simultaneously with ATP-TCA testing. ATP-TCA was performed as a routine procedure immediately following surgery. Viable ovarian cancer cells obtained from malignant tissues were tested for their sensitivity to paclitaxel (PTX; Corden Pharma Latina S.p.A., Sermoneta, Italy), carboplatin (CBP; Corden Pharma Latina S.p.A.), topotecan (TPT; Takara Biotechnology Co., Ltd., Dalian, China), gemcitabine (GEM; Eli Lilly and Company, Indianapolis, IN, USA), docetaxel (TXT; Aventis Pharma Ltd., Dagenham, UK), bleomycin (BLM; Nippon Kayaku Co. Ltd., Tokyo, Japan), etoposide (VP-16; Jiangsu Hengrui Medicine Co., Ltd., Jiangsu, China) and 4-hydroperoxycyclophosphamide (4-HC; Toronto Research Chemicals, Toronto, ON, Canada) using an in vitro ATP-TCA procedure.

In vitro ATP-TCA. Chemosensitivity was assessed in OEC tumor tissue samples using an ATP-TCA kit (Huzhou Haichuang Biotech Co., Ltd., Huzhou, Zhejiang, China), containing serum-free complete assay medium (CAM), digestive enzymes and luciferin-luciferase reagent. ATP-TCA tests were performed according to previously described methods $(18,19)$. Specimens $\left(1 \mathrm{~cm}^{3}\right)$ from solid tumors were obtained during surgery and cut into smaller fragments $\left(1 \mathrm{~mm}^{3}\right)$, which were then dissociated to prepare suspensions of single cells by incubation in 5-10 $\mathrm{ml}$ sterile digestive enzyme reagent for $2-3 \mathrm{~h}$ at $37^{\circ} \mathrm{C}$ in a $5 \% \mathrm{CO}_{2}$ incubator. Subsequent to adjusting the concentration of the cell suspension to $2-4 \times 10^{5} / \mathrm{ml}$, $100-\mu 1$ cell suspensions were added to each well of a 96-well polypropylene microplate. Single agents were tested at five different doses $(12.5,25,50,100$ and $200 \%)$ of a standard test drug concentration (TDC). The TDC values were $13.8 \mu \mathrm{g} / \mathrm{ml}$ for PTX, $25 \mu \mathrm{g} / \mathrm{ml}$ for CBP, $0.14 \mu \mathrm{g} / \mathrm{ml}$ for TPT, $25 \mu \mathrm{g} / \mathrm{ml}$ for GEM, $10 \mu \mathrm{g} / \mathrm{ml}$ for TXT, $3 \mu \mathrm{g} / \mathrm{ml}$ for $4-\mathrm{HC}, 0.6 \mu \mathrm{g} / \mathrm{ml}$ for BLM and $20 \mu \mathrm{g} / \mathrm{ml}$ for VP-16. For each concentration, two wells were used as controls, one containing $100 \mu \mathrm{l}$ ATP inhibitor for maximum inhibition (positive control) and the other containing CAM only (no drug, negative control). Plates were incubated for 5-6 days at $37^{\circ} \mathrm{C}$ with $95 \%$ humidity in a $5 \% \mathrm{CO}_{2}$ incubator. Following incubation, the cells were lysed by the addition of $50 \mu \mathrm{l}$ ATP extraction reagent, and $50 \mu \mathrm{l}$ luciferin-luciferase reagent was added to each well. Measurements of luminescence were recorded using a microplate luminometer (Orion II; Berthold Diagnostic Systems, Bad Wildbad, Germany), and inhibition curves were established.

Data analysis. Data were exported to an Excel spreadsheet (2010; Microsoft, Redmond, WA, USA), and the results were interpreted and compared using the parameters $\mathrm{IC}_{50}, \mathrm{IC}_{90}$ and SI (SI = 500 - sum of \% TGI at 200, 100, 50, 25 and $12.5 \%$ TDC). Three categories of in vitro sensitivity were defined as follows: Sensitivity (S), $\mathrm{IC}_{90} \leq 100 \% \mathrm{TDC}$ and $\mathrm{IC}_{50}<25 \%$ TDC; weak sensitivity (WS), $\mathrm{IC}_{90} \leq 100 \%$ TDC and $\mathrm{IC}_{50}<25 \%$ $\mathrm{TDC}$ or $\mathrm{SI} \leq 250$; and resistance $(\mathrm{R}), \mathrm{SI}>250$. According to the current clinical criteria with regard to platinum resistance and sensitivity, clinical CBP-sensitive patients indicates those patients who achieved complete remission and experienced relapse six months or later following initial platinum-containing chemotherapy, whereas CBP-resistant patients are those who demonstrated recurrence within $<6$ months (20).

Quality controls for each assay were conducted as follows: The variability of individual ATP values was controlled by measuring each drug-treated sample twice. Samples with a coefficient of variation $(\mathrm{CV})>0.15$ were rejected and retested. In the present study, the average CV was 0.065 (range, 0.024-0.127).

Statistical analysis was performed using SPSS software (version 17.0 for Windows; SPSS, Inc., Chicago, IL, USA). Data are presented as the mean \pm standard deviation, and comparisons were made using Student's t-test, the $\chi^{2}$ test and an analysis of variance. The correlation analysis was performed using Spearman's rank correlation test. $\mathrm{P}<0.05$ was considered to indicate a statistically significant difference.

\section{Results}

In vitro results. The median age of the patients was 56.15 years (range, 23-79 years). All the specimens collected produced evaluable results (100\%), and the tumor characteristics of the samples were analyzed (Table I). The results revealed considerable heterogeneity in chemosensitivity among the tumor samples tested. There were significant differences between the mean SIs obtained using various agents (Fig. 1A). The agent with the lowest SI values was PTX (mean SI, 152.79), followed 
Table I. Characteristics of tumor samples $(n=80)$.

\begin{tabular}{lc}
\hline Characteristic & $\mathrm{n}(\%)$ \\
\hline Histology & \\
Serous & $51(63.8)$ \\
Mucinous & $4(5.0)$ \\
Clear cell & $13(16.2)$ \\
Endometrioid & $8(10.0)$ \\
Transitional cell & $4(5.0)$ \\
FIGO stage & \\
I & $8(10.0)$ \\
II & $9(11.2)$ \\
III & $63(78.8)$ \\
Grade of differentiation & \\
High & $6(7.5)$ \\
Mild & $10(12.5)$ \\
Low & $64(80.0)$ \\
Primary & $53(66.2)$ \\
Recurrent & $27(33.8)$ \\
\hline
\end{tabular}
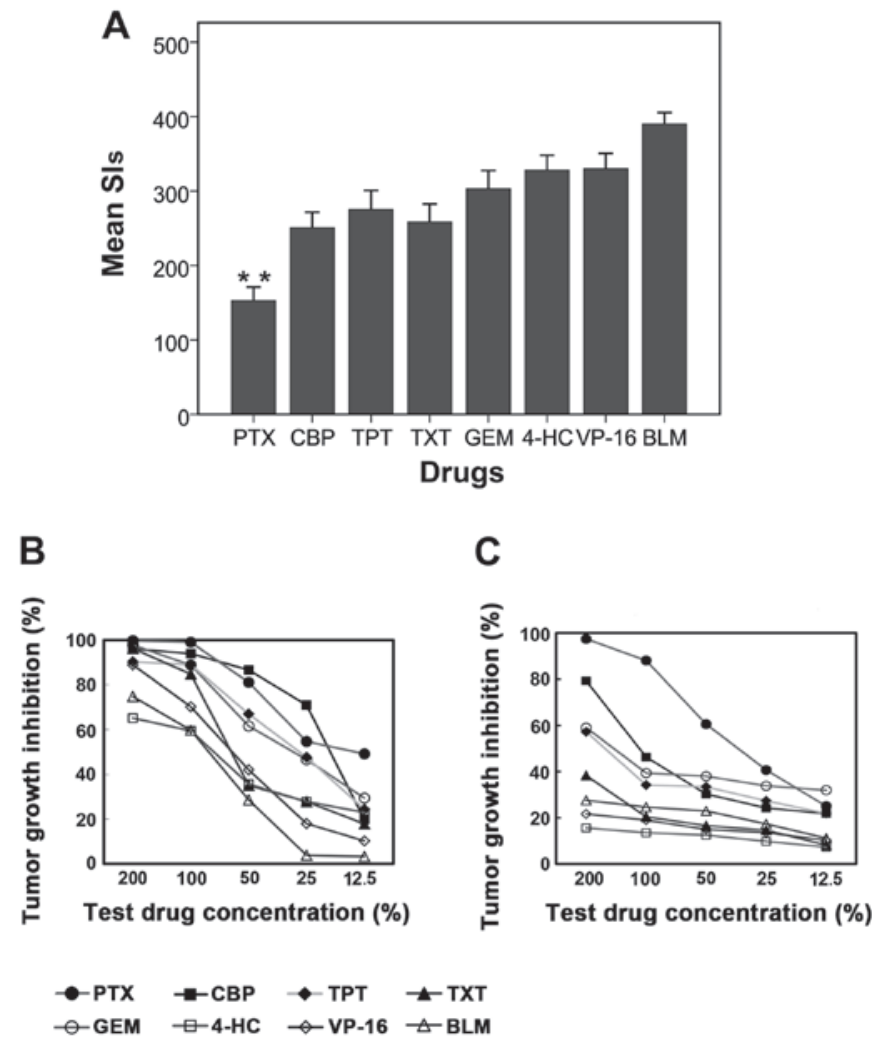

Figure 1 . Heterogeneity in chemosensitivity among the ovarian epithelial cancer specimens tested. (A) Frequency histograms (mean+95\% confidence interval) demonstrating substantial heterogeneity of the sensitivity indices (SIs) among the chemotherapy agents tested; (B and C) tumor growth inhibition curves from two ovarian tumor specimens of the same stage, and with the same histological features and grade of differentiation, demonstrating considerable heterogeneity in sensitivity to the same chemotherapy agents. The $\mathrm{X}$-axis is the test drug concentration percentage, and the Y-axis is the tumor growth inhibition percentage. (B) The results of a specimen with higher chemosensitivity and (C) the results of a specimen with lower chemosensitivity. PTX, paclitaxel; CBP, carboplatin; TPT, topotecan; TXT, docetaxel; GEM, gemcitabine; 4-HC, 4-hydroperoxycyclophosphamide; VP-16, etoposide; BLM, bleomycin. ${ }^{* *} \mathrm{P}<0.01$ vs. CBP, TXT, TPT, GEM, 4-HC, VP-16 or BLM.
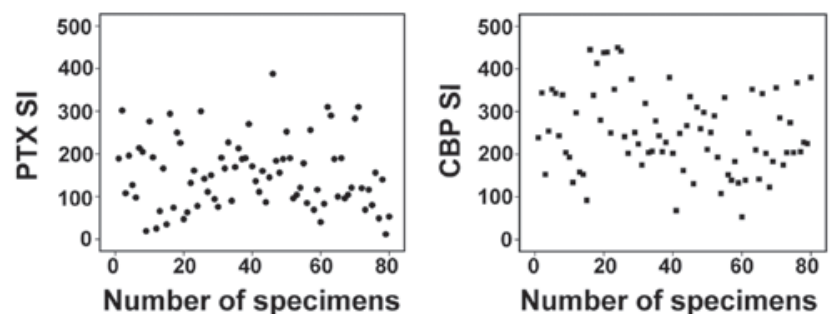

Number of specimens
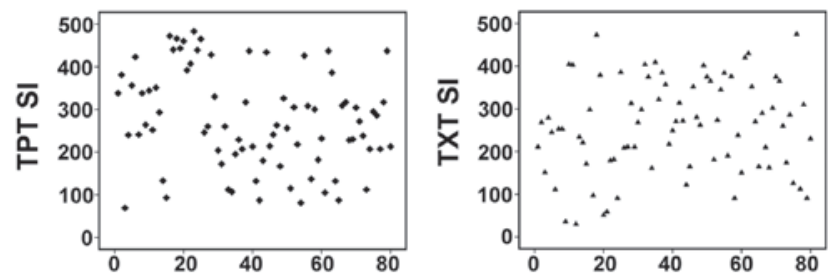

Number of specimens

Number of specimens
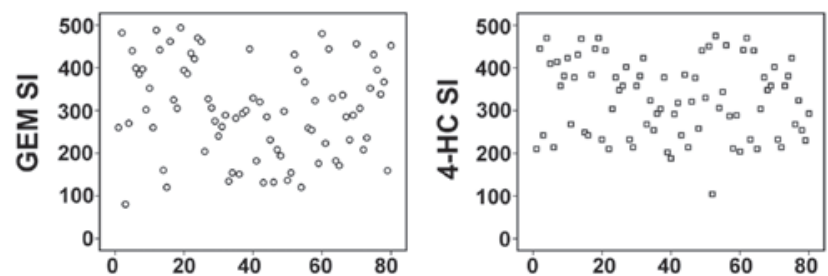

Number of specimens

Number of specimens
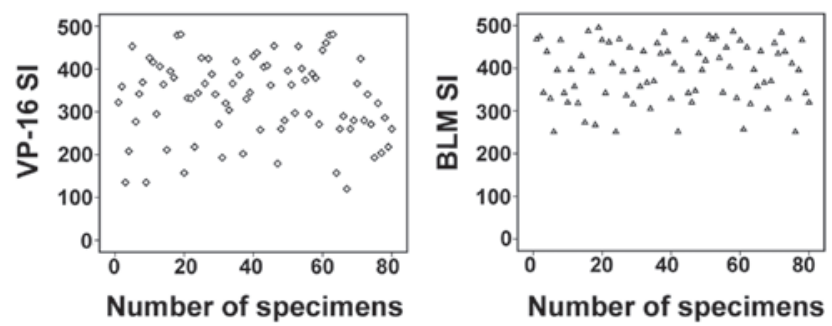

Figure 2. Scatter plots demonstrating heterogeneity of the tested chemotherapy agents in various tumor samples. SI, sensitivity index; PTX, paclitaxel; CBP, carboplatin; TPT, topotecan; TXT, docetaxel; GEM, gemcitabine; 4-HC, 4-hydroperoxycyclophosphamide; VP-16, etoposide; BLM, bleomycin.

by CBP (mean SI, 250.69), TXT (mean SI, 258.34) and TPT (mean SI, 275.29). The agent with the highest SIs was BLM (mean SI, 390.04).

The differences in SIs were observed between specimens of the same stage, and with the same histological features and grade of differentiation (Fig. 1B and C). There were also distinct differences among the SIs of the 80 OEC specimens obtained using the same drugs (Fig. 2). Overall, the sensitivity of the tumors to the agents tested was in the following order: PTX $>$ $\mathrm{CBP}>\mathrm{TPT}>\mathrm{TXT}>\mathrm{GEM}>4$-HC $>$ VP-16 > BLM (Table II).

In samples from recurrent ovarian cancer, the sensitivity rates for PTX and CBP were 85.7\% (24/28) and 60.7\% (17/28), respectively. Analysis of the clinical data revealed that all 17 recurrent specimens sensitive to CBP in vitro experienced a relapse at six months or later following initial platinum-containing chemotherapy and achieved complete remission, whereas the 11 recurrent specimens resistant to $\mathrm{CBP}$ in vitro experienced recurrence within the first six months following initial treatment.

Correlation of ATP-TCA with clinical sensitivity/resistance results. All cases were followed up for at least six months 
Table II. Results of chemosensitivity assays in ovarian epithelial cancer samples.

\begin{tabular}{|c|c|c|c|c|}
\hline \multirow[b]{2}{*}{ Drug } & \multicolumn{3}{|c|}{ No. $(\%)$} & \multirow[b]{2}{*}{ Sensitivity assessed, $\%$} \\
\hline & $\mathrm{S}$ & WS & $\mathrm{R}$ & \\
\hline PTX & $44(55.0)$ & $22(27.5)$ & $14(17.5)$ & 82.5 \\
\hline CBP & $21(26.4)$ & $26(32.4)$ & $33(41.2)$ & 58.8 \\
\hline $\mathrm{TPT}$ & $14(17.5)$ & $23(28.7)$ & $43(53.8)$ & 46.2 \\
\hline TXT & $18(22.5)$ & $18(22.5)$ & $44(55.0)$ & 45.0 \\
\hline GEM & $13(16.3)$ & $13(16.3)$ & $54(67.5)$ & 32.5 \\
\hline 4-HC & $3(3.7)$ & $18(22.5)$ & $59(73.8)$ & 26.2 \\
\hline VP-16 & $4(6.3)$ & $10(11.2)$ & $66(82.5)$ & 17.5 \\
\hline BLM & $0(0.0)$ & $0(0.0)$ & $80(100.0)$ & 0.0 \\
\hline
\end{tabular}

S, sensitivity; WS, weak sensitivity; R, resistance; PTX, paclitaxel; CBP, carboplatin; TPT, topotecan; TXT, docetaxel; GEM, gemcitabine; 4-HC, 4-hydroperoxycyclophosphamide; VP-16, etoposide; BLM, bleomycin.

Table III. Sensitivity, specificity, PPV and NPV at different SI cut-off points.

\begin{tabular}{lccc}
\hline SI cut-off point & Sensitivity, $\%$ & Specificity, $\%$ & PPV, $\%$ \\
\hline 250 & 88.6 & 77.8 & 83.0 \\
300 & 88.6 & 50.0 & 68.0 \\
200 & 43.0 & 91.6 & 86.0 \\
\hline
\end{tabular}

SI, sensitivity index; PPV, positive predictive value; NPV, negative predictive value.

Table IV. Results of correlation analysis between pairs of chemotherapy drugs tested.

\begin{tabular}{lcr}
\hline Drug & Correlation coefficient, $r$ & P-value \\
\hline PTX and TXT & 0.701 & $0.000^{\mathrm{a}}$ \\
CBP and TPT & 0.686 & $0.000^{\mathrm{a}}$ \\
TPT and GEM & 0.660 & $0.000^{\mathrm{a}}$ \\
CBP and GEM & 0.563 & $0.000^{\mathrm{a}}$ \\
TXT and VP16 & 0.422 & $0.000^{\mathrm{a}}$ \\
\hline
\end{tabular}

PTX, paclitaxel; TXT, docetaxel; CBP, carboplatin; TPT, topotecan; GEM, gemcitabine; VP-16, etoposide. ${ }^{\text {a }}<0.01$.

following initial chemotherapy. A total of 44 patients $(55 \%)$ were classified as clinical CBP-sensitive and 36 patients (45\%) as clinical CBP-resistant. The assay demonstrated a sensitivity, specificity, positive predictive value (PPV) and negative predictive value (NPV) of $88.6,77.8,83$ and $84.8 \%$, respectively, at SI 250. The sensitivity, specificity, PPV and NPV at SI 200 and SI 300 were also tested, and the results revealed a distinct advantage when choosing 250 as the cut-off point of SI (Table III).

Correlation analysis between pairs of chemotherapy drugs tested. The correlation analysis among the SIs of all eight drugs was performed using Spearman's rank correlation test. The results revealed positive correlations between several drugs. There were significant correlations between PTX and TXT $(\mathrm{P}<0.001)$ and among CBP, TPT and GEM $(\mathrm{P}<0.001)$ (Table IV).

Correlation between the ATP-TCA results and clinical indicators of tumor samples. An association analysis between the ATP-TCA results and the stage or differentiation grade of samples was performed using the $\chi^{2}$ test. The results revealed that early-stage (I/II) or high- to mildly-differentiated OEC specimens had lower chemosensitivity to PTX or CBP than advanced-stage (III) or low-differentiated specimens, respectively (Table V). The SIs with different stages or differentiation grades of samples also demonstrated distinct differences (Fig. 3). 
Table V. Correlation between ATP-TCA results for PTX or CBP and the stage or grade of differentiation of tumor samples.

\begin{tabular}{|c|c|c|c|c|c|c|}
\hline \multirow[b]{2}{*}{ Drug } & \multicolumn{3}{|c|}{ FIGO stage } & \multicolumn{3}{|c|}{ Grade of differentiation } \\
\hline & $\mathrm{I} / \mathrm{II}, \mathrm{n}$ & III, n & P-value & High-mild, $\mathrm{n}$ & Low, $\mathrm{n}$ & P-value \\
\hline \multicolumn{7}{|l|}{ PTX } \\
\hline $\mathrm{S}+\mathrm{WS}$ & 9 & 57 & $0.001^{\mathrm{a}}$ & 8 & 58 & $0.001^{\mathrm{a}}$ \\
\hline $\mathrm{R}$ & 8 & 6 & & 8 & 6 & \\
\hline \multicolumn{7}{|l|}{ CBP } \\
\hline $\mathrm{S}+\mathrm{WS}$ & 5 & 42 & $0.007^{\mathrm{a}}$ & 6 & 41 & $0.05^{\mathrm{b}}$ \\
\hline $\mathrm{R}$ & 12 & 21 & & 10 & 23 & \\
\hline
\end{tabular}

S, sensitivity; WS, weak sensitivity; R, resistant; PTX, paclitaxel; CBP, carboplatin. ${ }^{\mathrm{a}} \mathrm{P}<0.01$ and ${ }^{\mathrm{b}} \mathrm{P} \leq 0.05$.

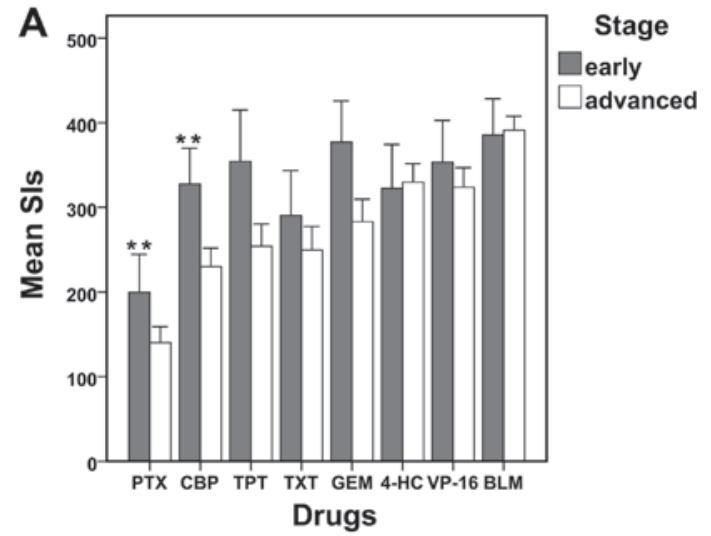

B

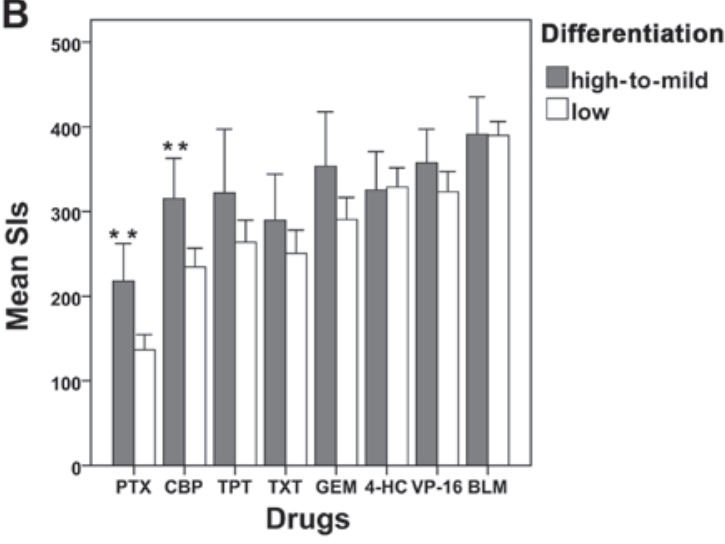

Figure 3. Frequency histograms (mean+95\% confidence interval) of the sensitivity indices (SIs) in specimens with different stages or differentiation grades among the chemotherapy agents tested. (A) Different stages. The SIs values of PTX or CBP in early-stage (I/II) OEC specimens are significant higher than advanced-stage (III) specimens. ${ }^{* *} \mathrm{P}<0.01$ vs. advanced-stage (III) specimens. (B) Different differentiation grades. The SIs values of PTX or CBP in high-to mildly-differentiated OEC specimens are significant higher than low-differentiated specimens. ${ }^{* *} \mathrm{P}<0.01$ vs. low-differentiated specimens. PTX, paclitaxel; CBP, carboplatin; TPT, topotecan; TXT, docetaxel; GEM, gemcitabine; 4-HC, 4-hydroperoxycyclophosphamide; VP-16, etoposide; BLM, bleomycin.

\section{Discussion}

In the present study, the heterogeneity in OECs was evaluated using an in vitro ATP-TCA, which has been widely used for determining the drug sensitivity rates of solid tumors, and reliable results were obtained. To ensure the accuracy of the results, the reasonable SI cut-off point was determined by comparing the in vitro drug sensitivity and clinical sensitivity at various SI values. It was observed that the sensitivity, specificity, PPV and NPV of ATP-TCA were better at SI 250, which was consistent with previous studies (12-14). The results indicated that ATP-TCA is a reliable in vitro drug sensitivity testing method and that the suitable cut-off point of SI is 250.

The present results demonstrated that there was considerable heterogeneity in chemosensitivity between the OEC samples tested, even between specimens of the same pathological type, clinical stage and tumor classification, which may be the basis for examining the mechanism of chemotherapy resistance in ovarian cancer. The present study investigated the differentiated tumor response to eight chemotherapeutic drugs when dividing the OEC specimens into recurrent or primary, early-stage (I/II) or advanced-stage (III), high- to mildly-differentiated or low-differentiated cases. PTX demonstrated the highest sensitivity of all agents tested $(82.5 \%$ in all specimens, $85.7 \%$ in recurrent specimens), followed by CBP (58.8 and $60.7 \%$, respectively), then TPT, TXT, GEM, 4-HC and VP-16. All specimens were resistant to BLM.

Analysis of the clinical data of recurrent OEC specimens revealed the correlation of chemotherapy resistance and recurrence interval following initial chemotherapy. All the clinical platinum-sensitive recurrent specimens were also sensitive to $\mathrm{CBP}$ in vitro, whereas the clinical platinum-resistant recurrent specimens were resistant to CBP in vitro, and the majority of the recurrent specimens were sensitive to PTX in vitro, which is consistent with current clinical criteria with regard to recurrent ovarian cancers. The 2014 National Comprehensive Cancer Network (NCCN) Clinical Practice Guidelines for ovarian cancer state that combination platinum-based chemotherapy is the preferred treatment following the first recurrence in platinum-sensitive patients (20-22). The guidelines also indicate that altering the schedule of PTX administration may produce secondary responses to treat platinum-resistant ovarian cancer, such as weekly single-agent PTX administration $\left(80 \mathrm{mg} / \mathrm{m}^{2} /\right.$ week $)$ or combined treatment with CBP (AUC 3/week) and PTX (70 mg/m²/week) $(23,24)$. In 2006, Kyrgiou et al (25) used multiple-treatment meta-analysis methodologies to analyze 198 clinical trials from the previous 40 years, and observed that CBP and PTX are not only the best 
first-line chemotherapy agents, but that they are also the best option as second-line drugs. The present results revealed the relatively high efficiency of PTX and CBP in recurrent ovarian cancers, particularly PTX, indicating the potential effectiveness of PTX retreatment in recurrent ovarian cancer. A highly significant correlation was also identified between PTX and TXT sensitivity, in addition to correlations between CBP, TPT and GEM treatments. These results highlight the significance of considering potential correlations among drug sensitivities to avoid potentially ineffective therapy and additional adverse effects when selecting appropriate drug regimens.

Prognoses of early-stage ovarian cancer are generally good, but responses to chemotherapy vary considerably in advanced-stage disease. According to NCCN guidelines, post-operative chemotherapy is not recommended for ovarian cancer patients with stage IA or IB, grade 1 disease. These patients demonstrate good survival rates with surgical treatment alone, whereas adjuvant chemotherapy is recommended for all other patients. Several studies have identified age, capsular rupture, histological subtype, stage, grade and positive cytology results as prognostic factors for recurrence and survival in early-stage ovarian cancers (26-30), and other studies have reported improved survival for adjuvant treatment in early-stage patients with high-risk factors (31). Swart et al (32) analyzed long-term follow-up data of patients with early-stage ovarian cancer and reported a significant benefit of adjuvant chemotherapy. In the adjuvant chemotherapy group, the 10-year absolute recurrence-free and overall survival rates demonstrated improvement compared with those for patients receiving no post-surgical chemotherapy (from 57 to $67 \%$ and from 64 to $72 \%$, respectively). However, Swart et al (32) also indicated that adjuvant chemotherapy reduced the risk of recurrence/ mortality or mortality alone in high-risk patients, but not in the low-risk group. Chan et al (33) analyzed 74 patients with recurrent early-stage, high-risk ovarian cancer (stages IA-IB, grade 3; stages IC and II, all grades; and clear cell carcinomas stages I-II), and observed that survival following recurrence was poor and comparable with that of patients with recurrent advanced-stage disease; the study recommended developing novel therapeutic modalities for these high-risk patients.

In the present study, it was observed that sensitivity to PTX or CBP in early-stage, high- to mildly-differentiated ovarian cancer specimens was remarkably lower than that of advanced stage or low-differentiated specimens. This finding was consistent with previous studies and suggests the poor effectiveness of chemotherapy following early-stage ovarian cancer recurrence. However, due to the limitations of the number of cases, further research to determine the characteristics associated with the response of early-stage ovarian cancer to chemotherapy is required. These results highlight the significance of developing more comprehensive initial treatment programs for early-stage ovarian cancer, particularly in patients with novel high-risk factors associated with early-onset disease, including the presence of BRCA1 and BRCA2 mutations or in families affected by Lynch syndrome $(34,35)$. The use of in vitro sensitivity testing methods may be an effective way to identify suitable agents for treating high-risk, early-stage ovarian cancer patients.
Thus, the results of the present study demonstrated the notable heterogeneity in chemosensitivity among specimens from ovarian cancer patients. This heterogeneity was to be associated with differing gene expression among individuals (6). Further studies are required to analyze differences in gene expression in ovarian cancer specimens and explore the mechanisms underlying chemotherapy resistance. ATP-TCA should be considered as an effective method for guiding the choice of chemotherapy drugs, avoiding ineffective treatment regimens, and investigating novel chemotherapy agents to improve patient prognosis and increase ovarian cancer survival.

\section{Acknowledgements}

This study was supported by the Capital Health Research and Development Projects of China (grant no. 2011-2008-05).

\section{References}

1. Jemal A, Siegel R, Xu J and Ward E: Cancer statistics, 2010. CA Cancer J Clin 60: 277-300, 2010.

2. Armstrong DK and Brady MF: Intraperitoneal therapy for ovarian cancer: a treatment ready for prime time. J Clin Oncol 24: 4531-4533, 2006.

3. Gore M, du Bois A and Vergote I: Intraperitoneal chemotherapy in ovarian cancer remains experimental. J Clin Oncol 24: 4528-4530, 2006.

4. Bao B, Ahmad A, Azmi AS, Ali S and Sarkar FH: Overview of cancer stem cells (CSCs) and mechanisms of their regulation: implications for cancer therapy. Curr Protoc Pharmacol 14: Unit 14.25, 2013.

5. Marusyk A and Polyak K: Tumor heterogeneity: causes and consequences. Biochim Biophys Acta 1805: 105-117, 2010.

6. Glaysher S, Gabriel FG, Johnson P, et al: Molecular basis of chemosensitivity of platinum pre-treated ovarian cancer to chemotherapy. Br J Cancer 103: 656-662, 2010.

7. Meinhold-Heerlein I and Hauptmann S: The heterogeneity of ovarian cancer. Arch Gynecol Obstet 289: 237-239, 2014.

8. Glaysher S and Cree IA: Cell sensitivity assays: the ATP-based tumor chemosensitivity assay. Methods Mol Biol 731: 247-257, 2011.

9. Kurbacher CM and Cree IA: Chemosensitivity testing using microplate adenosine triphosphate-based luminescence measurements. Methods Mol Med 110: 101-120, 2005.

10. Qi CJ, Ning YL, Zhu YL, et al: In vitro chemosensitivity in breast cancer using ATP-tumor chemosensitivity assay. Arch Pharm Res 32: 1737-1742, 2009.

11. Sevin BU, Peng ZL, Perras JP, et al: Application of an ATP-bioluminescence assay in human tumor chemosensitivity testing. Gynecol Oncol 31: 191-204, 1988.

12. Konecny G, Crohns C, Pegram M, et al: Correlation of drug response with the ATP tumor chemosensitivity assay in primary FIGO stage III ovarian cancer. Gynecol Oncol 77: 258-263, 2000

13. Neubauer H, Stefanova M, Solomayer E, et al: Predicting resistance to platinum-containing chemotherapy with the ATP tumor chemosensitivity assay in primary ovarian cancer. Anticancer Res 28: 949-955, 2008.

14. Zhao D, Zhang W, Li XG, et al: Predicting clinical chemo-sensitivity of primary ovarian cancer using adenosine triphosphate-tumor chemosensitivity assay combined with detection of drug resistance genes. Zhonghua Fu Chan $\mathrm{Ke} \mathrm{Za}$ Zhi 46: 193-198, 2011 (In Chinese).

15. Cree IA, Kurbacher CM, Lamont A, et al: A prospective randomized controlled trial of tumour chemosensitivity assay directed chemotherapy versus physician's choice in patients with recurrent platinum-resistant ovarian cancer. Anticancer Drugs 18: 1093-1101, 2007.

16. Sharma S, Neale MH, Di Nicolantonio F, et al: Outcome of ATP-based tumor chemosensitivity assay directed chemotherapy in heavily pre-treated recurrent ovarian carcinoma. BMC Cancer 3: 19, 2003.

17. Cree IA, Kurbacher CM, Untch M, et al: Correlation of clinical response to chemotherapy in breast cancer with ex vivo chemosensitivity. Anticancer Drugs 7: 630-635, 1996. 
18. Andreotti PE, Cree IA, Kurbacher CM, et al: Chemosensitivity testing of human tumors using a microplate adenosine triphosphate luminescence assay: clinical correlation for cisplatin resistance of ovarian carcinoma. Cancer Res 55: 5276-5282, 1995.

19. Ling ZQ, Qi CJ, Lu XX, et al: Heterogeneity of chemosensitivity in esophageal cancer using ATP-tumor chemosensitivity assay. Acta Pharmacol Sin 33: 401-406, 2012.

20. National Comprehensive Cancer Network: Ovarian cancer including fallopian tube cancer and primary peritoneal cancer. Version 1. 2013 http://www.nccn.org/professionals/physician_ gls/f_guidelines.asp\#ovarian Accessed February 13, 2015.

21. Fung-Kee-Fung M, Oliver T, Elit L, et al: Optimal chemotherapy treatment for women with recurrent ovarian cancer. Curr Oncol 14 195-208, 2007.

22. Parmar MK, Ledermann JA, Colombo N, et al: Paclitaxel plus platinum-based chemotherapy versus conventional platinum-based chemotherapy in women with relapsed ovarian cancer: the ICON4/ AGO-OVAR-2.2 trial. Lancet 361: 2099-2106, 2003.

23. Gynecologic Oncology Group, Markman M, Blessing J, Rubin SC, Connor J, Hanjani P and Waggoner S: Phase II trial of weekly paclitaxel $(80 \mathrm{mg} / \mathrm{m} 2)$ in platinum and paclitaxel-resistant ovarian and primary peritoneal cancers: a Gynecologic Oncology Group study. Gynecol Oncol 101: 436-440, 2006.

24. Sharma R, Graham J, Mitchell H, Brooks A, Blagden S and Gabra H: Extended weekly dose-dense paclitaxel/carboplatin is feasible and active in heavily pre-treated platinum-resistant recurrent ovarian cancer. Br J Cancer 100: 707-712, 2009.

25. Kyrgiou M, Salanti G, Pavlidis N, Paraskevaidis E and Ioannidis JP: Survival benefits with diverse chemotherapy regimens for ovarian cancer: meta-analysis of multiple treatments. J Natl Cancer Inst 98 $1655-1663,2006$

26. Vergote I: Prognostic factors in stage I ovarian carcinoma. Verh K Acad Geneeskd Belg 63: 257-271, 2001.

27. Gadducci A, Cosio S, Zola P, Sostegni B, Fuso L and Sartori E: Prognostic factors and clinical outcome of patients with recurrent early-stage epithelial ovarian cancer: an Italian multicenter retrospective study. Int J Gynecol Cancer 23: 461-468, 2013.
28. Seidman JD, Yemelyanova AV, Khedmati F, et al: Prognostic factors for stage I ovarian carcinoma. Int J Gynecol Pathol 29: 1-7, 2010.

29. Chan JK, Tian C, Monk BJ, et al: Prognostic factors for high-risk early-stage epithelial ovarian cancer: a Gynecologic Oncology Group study. Cancer 112: 2202-2210, 2008.

30. Tropé C, Kaern J, Hogberg T, et al: Randomized study on adjuvant chemotherapy in stage I high-risk ovarian cancer with evaluation of DNA-ploidy as prognostic instrument. Ann Oncol 11: 281-288, 2000

31. Bertelsen K, Hølund B, Andersen JE, Nielsen K, Strøyer I and Ladehoff P: Prognostic factors and adjuvant treatment in early epithelial ovarian cancer. Int J Gynecol Cancer 3: 211-218, 1993.

32. Swart AC on behalf of ICON collaborators: Long-term follow-up of women enrolled in a randomized trial of adjuvant chemotherapy for early stage ovarian cancer (ICON1). J Clin Oncol, ASCO Annual Meeting Proceedings Part I 25 (June 20 Supplement): 5509, 2007

33. Chan JK, Tian C, Teoh D, et al: Survival after recurrence is poor for women with early-stage high-risk ovarian cancer: a Gynecologic Oncology Group study. Gynecol Oncol 116: 307-311, 2010

34. Sinilnikova OM, Mazoyer S, Bonnardel C, et al: BRCA1 and BRCA2 mutations in breast and ovarian cancer syndrome: reflection on the Creighton University historical series of high risk families. Fam Cancer 5: 15-20, 2006.

35. Daly MB, Axilbund JE, Buys S, et al: Genetic/familial high-risk assessment: breast and ovarian. J Natl Compr Canc Netw 8: 562-594, 2010 